\title{
INOS Dimerization Inhibitor ASP9853
}

National Cancer Institute

\section{Source}

National Cancer Institute. iNOS Dimerization Inhibitor ASP9853. NCI Thesaurus. Code C116728.

An orally bioavailable small molecule inhibitor of inducible nitric oxide synthase (iNOS) with potential antineoplastic activity. Upon administration, ASP9853 inhibits iNOS dimerization, which results in decreased nitric oxide (NO) production. iNOS expression is upregulated in certain cancers and may invoke a chronic inflammatory state in tumor cells that promotes metastatic growth. 